\title{
DECISION TREE INDUCTION FROM COUNTEREXAMPLES
}

\author{
Nicolas Cebron, Fabian Richter and Rainer Lienhart \\ Multimedia Computing Lab, University of Augsburg, Universitaetsstr. 6a, Augsburg, Germany
}

Keywords: Decision trees, Counterexamples, Machine learning, Data mining, Decision making.

\begin{abstract}
While it is well accepted in human learning to learn from counterexamples or mistakes, classic machine learning algorithms still focus only on correctly labeled training examples. We replace this rigid paradigm by using complementary probabilities to describe the probability that a certain class does not occur. Based on the complementary probabilities, we design a decision tree algorithm that learns from counterexamples. In a classification problem with $K$ classes, $K-1$ counterexamples correspond to one correctly labeled training example. We demonstrate that even when only a partial amount of counterexamples is available, we can still obtain good performance.
\end{abstract}

\section{INTRODUCTION}

The goal of supervised classification is to deduce a function from examples in a dataset that maps input objects to desired outputs. By using a set of labeled training examples, we can train a classifier that can be used to predict the nominal target variable for unseen test data. To achieve this, the learner has to generalize from the presented data to unseen situations. While a plethora of algorithms for supervised classification has been developed, only a few works deviate from this classical setting.

In this paper, we focus our attention on decision trees. Especially in multi-class problems, they are a reliable and effective technique. They usually perform well and offer a simple representation in form of a tree or a set of rules that can be deduced from it. They have been used a lot in situations where a decision must be made effectively and reliably, e.g. in medical decision making (Podgorelec et al., 2002). However, like all inductive methods in machine learning, the performance of this classifier is based on correctly labeled training examples. Finding the correct class label for an example when generating a training set for the classifier can be difficult - especially when there is a large number of possible classes. In the work of (Joshi et al., 2010), it has been shown that the human error rate and the time needed to find the correct label grows with the number of classes; at the same time the user distress increases. In some situations, it might not even be possible for the human expert to determine the correct class label out of ma- ny possible class labels. In a normal classification setting, we would have to ignore this example.

As an example, we stick to the domain of medical decision making, where we have two common situations in which the human expert has problems providing the correct class label:

1. Ambiguous Information: different class labels (e.g. diseases) may be possible, but there is a lack of information to explicitly choose one of them. For example, it is unclear whether a person with headache symptoms is suffering from a cold or has the flu (or another type of disease).

2. Rare Cases: the determination of the class label may be difficult because of missing expertise in a special field. For example, it may be difficult to classify rare (so-called orphan) diseases.

In this work, we want to introduce a new paradigm in supervised classification: we do not obtain the label information itself, but the labels of the classes that this example does not belong to. We call these examples counterexamples. For the preceding examples in medical decision making, it can be very easy to specify the diseases that are not likely (e.g. not typhlitis, not heartburn, etc. for a headache symptom) in order to narrow down the set of possible classes. We argue that in many real world settings, it is much easier for the human expert to provide a counterexample instead of determining the correct class label. This does not only apply to the domain of medical decision making, it is also true for many other domains like image, music or text classification. 
Within our new framework of classification with counterexamples, we can gain information from almost every example in a dataset. However, we keep our framework open and the information of examples and counterexamples can be included seamlessly. In a classification problem with $K$ disjoint classes, there is of course a loss of information induced from this setting, as we can expect to observe less than $K-1$ labels for each counterexample in practice ${ }^{1}$. The question that we aim to answer in this paper is: How much does this loss of information influence the resulting classification model?

To the best of our knowledge, this is the first work that considers feedback in the form of counterexamples in a multiclass setting. Some works have investigated negative feedback in the image retrieval process (Ashwin et al., 2001), (Mueller et al., 2000). As the retrieval process corresponds to a two-class problem, these works only share the general idea of a different form of feedback with this work. At first sight, our work seems to be related to the domain of multilabel classification (Tsoumakas and Katakis, 2007), where a mapping from an example to a set of class labels is sought. However, our goal is to predict one class label from the set of counterexamples.

In order to quantify the information from counterexamples, we introduce the probability theory for counterexamples in section 2 . In section 3 , we will introduce the decision tree learning algorithm for counterexamples. We will present results on different benchmark datasets in section 4 and finally draw conclusion in section 5 .

\section{COUNTEREXAMPLE PROBABILITIES}

We begin by recapitulating the basic laws of probability theory: the probability of an event is the fraction of times that the event occurs out of the total number of trials, in the limit that the total number of trials goes to infinity. In our case, the probabilities correspond to the events that a certain class occurs in a set of examples. We denote the probability for class $k$ by $p(k)$. By definition, the probabilities must lie in the interval $[0,1]$, and if the events are mutually exclusive and include all outcomes, their probabilities must sum to one:

$$
0 \leq p(k) \leq 1
$$

\footnotetext{
${ }^{1}$ If we would have $K-1$ labels, we could deduce the corresponding label directly.
}

$$
\sum_{k=1}^{K} p(k)=1
$$

In order to work with counterexamples and to quantify the amount of classes that are not contained in a set of examples, we need to define complementary probabilities. A complementary probability for event $k$, denoted by $\bar{p}(k)$ describes the probability that event $k$ does not occur in a set of examples. By definition, the probability that event $k$ does not occur is the sum of the probabilities of all other events that have occurred:

$$
\left.\bar{p}(k)=\sum_{j=1, j \neq k}^{K} p(j)\right)
$$

The relation between $p(k)$ and $\bar{p}(k)$ is defined as $p(k)=1-\bar{p}(k)$.

Like normal probabilities, $\bar{p}(k)$ must lie in the interval $[0,1]$. However, as the set of events is not mutually exclusive (a counterexample may have more than one class that it does not belong to), we need to adapt the restriction from equation 2 taking into account the definition of $\bar{p}(k)$ :

$$
\begin{aligned}
\sum_{k=1}^{K}(\bar{p}(k)) & =\sum_{k=1}^{K}\left[\sum_{j=1, j \neq k}^{K} p(j)\right] \\
& =\sum_{k=1}^{K}[1-p(k)] \\
& =K-\sum_{k=1}^{K} p(k) \\
& =K-1
\end{aligned}
$$

Having established the basic laws for complementary probabilities and rules to transform probabilities into complementary probabilities and vice versa, we can use them in the design of a decision tree that learns from counterexamples in the next section.

\section{DECISION TREE INDUCTION}

We assume that instead of having one class label for each example, we have a vector $\vec{y}=\left(y_{1}, \ldots, y_{K}\right)$, where each entry $y_{k} \in\{0,1\}$ indicates whether we know that this example does not belong to class $k$ (1) or that we do not have any information concerning class $k$ for this example (0).

The main difference between learning a tree from examples and learning a tree from counterexamples is the notion of purity of a data partition. Figure 1 illustrates the situation of learning a decision tree from counterexamples. Each partition can now con- 

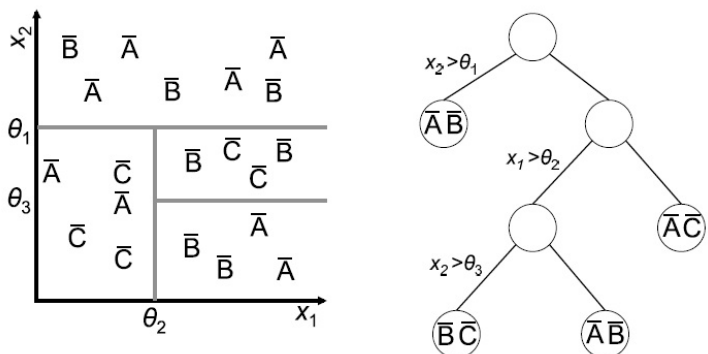

Figure 1: Decision tree with four partitions based on counterexamples.

tain multiple class labels of the classes that the examples do not belong to. The goal is to find partitions that have high complementary probabilities for $K-1$ classes as they correspond to a 'pure' distribution of the class label.

We use our definition of mapping from complementary probabilities to probabilities in section 2 to derive a new definition for the entropy (Shannon, 2001), which is commonly used to judge the quality of a data partition $X$ :

$$
\bar{H}(X)=\sum_{k=1}^{K}(1-\bar{p}(k)) \ln (1-\bar{p}(k)) .
$$

As can be seen in figure 1, the leaf nodes of our decision tree contain a distribution of complementary class probabilities. We can output this distribution or transform it to normal class probabilities and use the majority class as a decision.

\section{RESULTS}

The algorithms were implemented within the framework of the weka (Hall et al., 2009) and mulan software (Tsoumakas et al., 2011).

Each experiment has been been repeated 500 times. In each iteration, we split up the dataset randomly and use $30 \%$ for training and $70 \%$ for testing. We deduce the corresponding complementary class probabilities from the original class probabilities for each example. We then remove 0\% (corresponds to a fully labeled dataset) to $90 \%$ of information from the $\vec{y}$ vectors in the training dataset (plotted on the $\mathrm{x}$-axis) and plot the accuracy as a boxplot on the $y$-axis. As we remove information from the entries in $\vec{y}$, the counterexample probabilities $\bar{p}(k)$ become smaller. However, this is not an issue as $\bar{H}(X)$ scales monotonically with information removal as shown in the Appendix.

\subsection{Contact Lenses}

The lenses dataset consists of 24 examples. The goal is to predict whether a person should be fitted with hard or soft contact lenses or no contact lenses based on four attributes. Figure 2 shows the accuracy of the decision tree that is induced from counterexamples for a varying amount of information. We can ob-

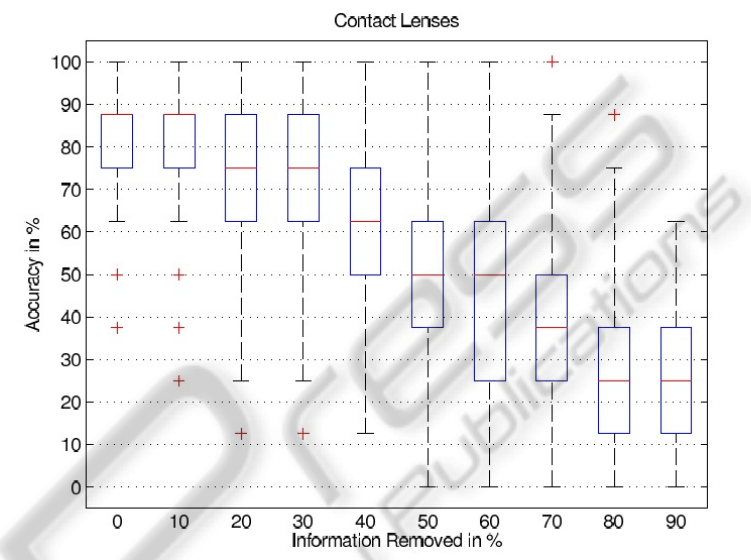

Figure 2: Information vs. accuracy on lenses dataset.

serve a linear decline of accuracy with the amount of information removed. As the dataset is very small, removing information has a deep impact on the resulting decision tree. However, if we remove up to $30 \%$ of information, we still get acceptable accuracy.

\subsection{Balance Scale}

In the balance scale dataset, each example is classified as having the balance scale tip to the right, tip to the left, or be balanced. The four attributes are the left weight, the left distance, the right weight, and the right distance. Figure 3 shows the accuracy of the decision tree that is induced from counterexamples for a varying amount of information. The decline in accuracy is not as steep as for the contact lenses dataset, which is due to the larger number of 187 examples in the training set.

\subsection{Nursery}

The nursery dataset was derived from a hierarchical decision model originally developed to rank applications for nursery schools in five different classes. It contains 12960 examples. As the accuracy does almost not decline between $0 \%$ and $99 \%$, we plot the experiment with $99 \%$ to $99.9 \%$ information removed in Figure 4. We can observe that the accuracy declines very late. 


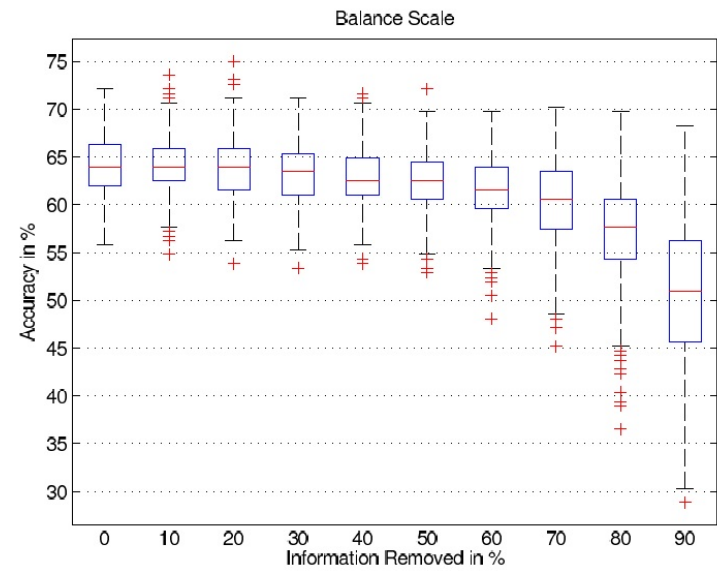

Figure 3: Information vs. accuracy on balance scale dataset.

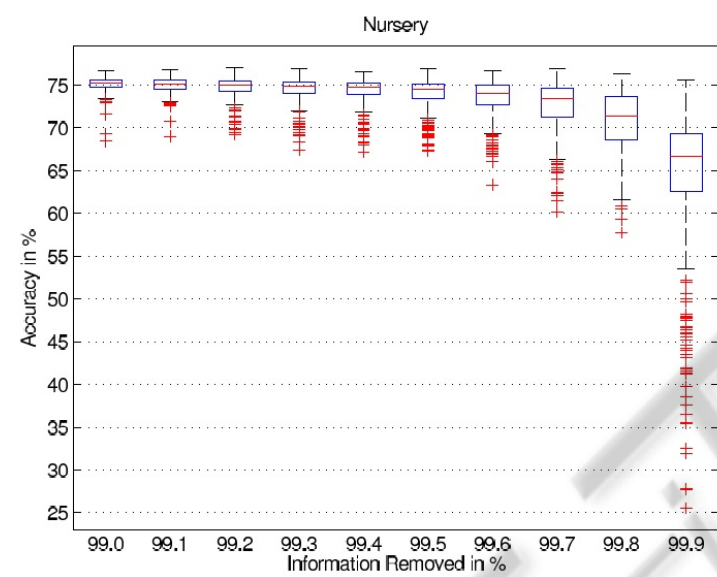

Figure 4: Informations vs. accuracy on nurse dataset.

\section{CONCLUSIONS}

In this work, we have presented a new approach to induce a decision tree classifier from counterexamples. Based on complementary probabilities we have adapted the entropy measure in order to work with this new type of human feedback. Normal examples can also be integrated seamlessly by deducing the complementary class probabilities from the given class probabilities. We have observed that this approach works well even if we remove a significant amount of information from the training examples. This shows that we can learn from counterexamples in a practical setting, where the user typically provides less than $K-1$ class labels. We hope that this work does inspire future work in the community on different forms of feedback in machine learning.

\section{REFERENCES}

Ashwin, T., Jain, N., and Ghosal, S. (2001). Improving image retrieval performance with negative relevance feedback. Acoustics, Speech, and Signal Processing, IEEE International Conference on, 3:1637-1640.

Hall, M., Frank, E., Holmes, G., Pfahringer, B., Reutemann, P., and Witten, I. H. (2009). The weka data mining software: an update. SIGKDD Explor. Newsl., 11:1018.

Joshi, A. J., Porikli, F., and Papanikolopoulos, N. (2010). Breaking the interactive bottleneck in multi-class classification with active selection and binary feedback. In CVPR, pages 2995-3002. IEEE.

Mueller, H., Mueller, W., Squire, D. M., Marchand-Maillet, S., and Pun, T. (2000). Strategies for positive and negative relevance feedback in image retrieval. In Proceedings of the International Conference on Pattern Recognition - Volume 1, volume 1, pages 1043-1046, Washington, DC, USA. IEEE Computer Society.

Podgorelec, V., Kokol, P., Stiglic, B., and Rozman, I. (2002). Decision trees: An overview and their use in medicine. J. Med. Syst., 26:445-463.

Shannon, C. E. (2001). A mathematical theory of communication. SIGMOBILE Mob. Comput. Commun. Rev., 5(1):3-55.

Tsoumakas, G. and Katakis, I. (2007). Multi label classification: An overview. International Journal of Data Warehouse and Mining, 3(3):1-13.

Tsoumakas, G., Spyromitros-Xioufis, E., Vilcek, J., and Vlahavas, I. (2011). Mulan: A java library for multilabel learning. Journal of Machine Learning Research. (to appear).

\section{APPENDIX}

We use the scalar $\alpha \geq 1$ to compensate for the decline in $\bar{p}(k)$ due to the information removal.

$$
\begin{aligned}
& \bar{H}(X)= \sum_{k=1}^{K} \alpha(1-\bar{p}(k)) \ln (\alpha(1-\bar{p}(k))) \\
&= \sum_{k=1}^{K} \alpha(1-\bar{p}(k))[\ln (\alpha)+\ln (1-\bar{p}(k))] \\
&= \alpha \sum_{k=1}^{K}(1-\bar{p}(k)) \ln ((1-\bar{p}(k))) \\
& \quad+\alpha \sum_{k=1}^{K}(1-\bar{p}(k)) \ln (\alpha) \\
&=\alpha \bar{H}(X)+\alpha \ln (\alpha) .
\end{aligned}
$$

